American Journal of Applied Sciences 9 (9): 1503-1511, 2012

ISSN 1546-9239

(C) 2012 Science Publication

\title{
Mulberry Fruits Protects Against Age-Related Cognitive Decline
}

\author{
${ }^{1,2}$ Jintanaporn Wattanathorn, ${ }^{1,2}$ Supaporn Muchimapura, \\ ${ }^{1,2}$ Wipawee Thukhammee, ${ }^{1,2}$ Terdthai Tong-un, ${ }^{1,2}$ Panakporn Wannanon, \\ ${ }^{1,3}$ Nattaporn Phunchago, ${ }^{1,3}$ Pratchaya Kaewkaen, ${ }^{4}$ Thanes Chantes, \\ ${ }^{4}$ Wiroje Kaewruang, ${ }^{4}$ Suttisan Pimpasalee and ${ }^{1,2}$ Sathaporn Wongareonwanakij \\ ${ }^{1}$ Integrative Complementary Alternative Medicine Research Center, \\ Khon Kaen University, Khon Kaen, Thailand \\ ${ }^{2}$ Department of Physiology, Faculty of Medicine, \\ Khon Kaen University, Khon Kaen, Thailand \\ ${ }^{3}$ Department of Physiology (Neuroscience Program), Faculty of Medicine, \\ Khon Kaen University, Khon Kaen, Thailand \\ ${ }^{4}$ Department of Sericulture, \\ The Queen Sirikit Ministry of Agriculture and Cooperatives, Thailand
}

\begin{abstract}
Problem statement: The phenolic compounds and anthocyanin contents, the antioxidant and Acetylcholinesterase Inhibitor (AChI) activities of mulberry fruits were determined. Approach: Analysis of phenolic compounds and anthocyanin were performed using Folin Ciocalteu reagent and pH-differential method respectively. The antioxidant activity was determined using DPPH and FRAP assays while AChI activity was determined using Ellman method. Results: The mulberry fruit powder contained high amount of phenolic compounds and anthocyanin, It also exhibited strong antioxidant and AChEI activities. Based on the beneficial effect of polyphenol compounds and anthocyanin on brain, we also further evaluated the neuroprotective and cognitive enhancing effects of mulberry fruits in animal model of age-related cognitive decline. Surprisingly mulberry fruits could mitigate brain damage and memory impairment in age-related cognitive decline. The possible mechanism might be associated with its antioxidant and AChEI activities. Conclusion: In conclusion, Mulberry fruits are the potential neuroprotectant and cognitive enhancer. However, further investigations are required.
\end{abstract}

Key words: Mulberry fruit, hippocampal damage, memory impairment, Senescence-Accelerated Mice (SAMP), Gallic Acid Equivalents (GAE), Fresh Weight (FW)

\section{INTRODUCTION}

At present, the average age increasing at an unprecedented rate. Demographic projections suggest that the number of people aged over 65 years will nearly triple by 2050 , accounting for $14 \%$ of the world (Lutz et al., 2008). Normal aging is reported to be associated with a slow decline in brain functions such as sensory and motor performance and at times, this decline is accompanied by progressive memory loss, dementia and cognitive dysfunctions, ultimately resulting in limited functionality. It has been reported that the cognitive impairment in both aged human and rodents is correlated with the accumulation of oxidative damage to lipids, proteins, nucleic acids (Butterfield et al., 2006; Forster et al., 1996; Murali and Panneerselvam, 2007) and the vulnerability of various neurotransmitters to oxidative stress (Govoni et al., 2010; Mokrasch and Teschke, 1984; Pradham, 1880). Moreover, it is also reported to be related to the hypofunction of cholinergic system and manifested by the decreased Acetylcholinesterase (AChE), one of the major markers of cholinergic function in various cerebral areas (Papandreou et al., 2006).

Recently, numerous researches clearly revealed that various antioxidant supplements and phytochemical components provided the beneficial for preserving brain functions and forestalling the age-related deficits (Shukitt-Hale et al., 2008). Thus, this raised the possibility that the substances possessing antioxidant activity should be able to protect against the brain area, contributing the important role on learning and memory resulting the improved memory impairment.

Corresponding Author: Jintanaporn Wattanathorn, Integrative Complementary Alternative Medicine Research Center, Khon Kaen University, Khon Kaen, Thailand 
Mulberry or Morus alba belongs to the family Moraceae. It has been widely planted in both the Northeast and North of Thailand. Mulberry fruit is widely regarded as a nutritious food. It can be eaten freshly or widely used in the production of wine, fruit juice, jam and canned food (Ning et al., 2005). Mulberry fruit is not only used as fruit but also as medicine. According to the traditional folklore, mulberry fruit is used to protect against liver and kidney damage, strengthen the joints, improve eyesight and have anti-aging effects (Shizhen, 2008). It is also used for the treatment of sore throat, fever, hypertension and anemia (Ma, 2002; Gong and Zhu, 2008). In addition, recent findings showed that mulberry fruits could protect against brain damage in various conditions including Parkinson's disease (Kim et al., 2010) and cerebral ischemia (Kang et al., 2006). Moreover, mulberry fruit extract also decreased $\beta$ amyloid protein and improved learning impairment in Senescence-Accelerated Mice (SAMP) (Shih et al., 2010). Although, several researches had already demonstrated the neuroprotective of mulberry fruits, less evidence about the effect of mulberry fruits on hippocampus, the area contributing important role on learning and memory, in the age-related cognitive impairment is available. Thus, this study was set up to elucidate this issue in animal model of age-related cognitive impairment induced by a cholinotoxin, AF64A.

\section{MATERIALS AND METHODS}

Preparation of mulberry fruits freeze-dried powder: All mulberry fruits (Morus alba var Chiangmai) used in this study is prepared and provided by The Institute of Queen Sirikit Seri Culture, Thailand. Mulberry fruits were collected from the Queen Sirikit Seri Culture Center Udon Thani. All berries were picked at the commercially ripen stage and selected according to uniformity color. Then, the fruits were dried at 70 degree celcius for 4 days and grounded to powder. In addition, the mulberry fruit powders were also kept in dark air tight bottle at $-20^{\circ} \mathrm{C}$.

Chemicals: Donepezil hydrochloride (Aricept $10 \mathrm{mg}$ tablet $^{-1}$ ) (Pfizer pharmaceuticals Inc.) was used as standard drugs in this study. They were dissolved in propylene glycol and administered via oral route. All chemical substances used in this study were analytical grade.

Determination of phenolic compound: Total TF content of the plant extracts were determined according to the well-established colorimetric assay (Meda et al., 2005). Gallic acid was used as a standard and the results were expressed as milligram Gallic Acid
Equivalents (GAE) per gram of dry matter. Finally, the data were converted to $\mathrm{mg} \mathrm{GAE} / \mathrm{g}$ fresh matter of fruit. Total phenolic compound was assessed both at 1 month of preparation and 1 year after preparation.

Total anthocyanin content: Anthocyanin quantification was performed by the $\mathrm{pH}$-differential method (Wrolstad et al., 2001). Calculation of the anthocyanins concentration was based on a cyanidin-3glucoside molar extinction coefficient 26,900 and a molecular mass of $449.2 \mathrm{~g} \mathrm{~mol}^{-1}$. Results were expressed as miligrams $(\mathrm{mg})$ of Cyanidin-3-Glucoside Equivalents (CGE) per $100 \mathrm{~g}$ of Fresh Weight (FW). Anthocyanin content was determined both at 1 month of prepparation and 1 year after preparation.

\section{Antioxidant capacity:}

DPPH radical scavenging assay: Radical scavenging capacity was determined according to the method outlined by Scapagnini et al. (2010). A calibration curve was prepared, using Trolox (6-hydroxy-2,5,7,8tetramethylchromane-2-carboxylic acid) and the results were expressed as mmol Trolox equivalents per $100 \mathrm{~g}$ of fresh weight(mmol TEAC/100 $\mathrm{g} \mathrm{FW})$.

Ferric reducing/antioxidant power assay (FRAP assay): The FRAP assay was used to estimate the reducing capacity of tested substances, according to the original method of Benzie and Strain (1996). A calibration curve was prepared, using an aqueous solution of ferrous sulphate $\mathrm{FeSO} 4.7 \mathrm{H} 2 \mathrm{O}$ and the results, obtained from three replicate extractions, were expressed as mmol $\mathrm{FeSO}_{4} \cdot 7 \mathrm{H} 2 \mathrm{O}$ per $100 \mathrm{~g}$ of fresh weight $\left(\mathrm{mmol} \mathrm{Fe}^{2+} / 100 \mathrm{~g} \mathrm{FW}\right)$.

Determination of Acetylcholinesterase (AChE): Inhibition of $\mathrm{AChE}$ was evaluated according to the method Ellman et al. (1961) with some modification. The assay is based on the spectrophotometric measurement of the increased yellow color produced from thiocholine when it reacts with the dithiobisnitrobenzoate ion. Rivastigmine was used as a positive control and water served as a positive control. The increase in absorbance value due to the spontaneous hydrolysis of the substrate was corrected by subtracting the ratio of the reaction before adding the enzyme from the rate after the enzyme addition. Percentage inhibition by extracts and galanthamine were calculated using the equation below.

Inhibition $(\%)=\frac{[1-\text { Sample reaction rate }]}{\text { Blank reaction rate }}$

Animals: In vivo experiments were carried out on male Wistar rats, weighing 180-200 g, that were housed four per cage under a $12 \mathrm{~h}$ light/dark cycle in a room with 
controlled temperature $\left(22 \pm 1^{\circ} \mathrm{C}\right)$. Food and water were available ad libitum. Animals were handled daily (between 9:00 and 11:00 A.M) 5 days before the experiment in order to adapt them to manipulation and minimize non-specific stress responses. Rats were randomly allocated into several experimental groups, each comprising 8 animals. All experiments followed the guidelines on ethical standard for investigation of experimental pain in animals and approved by the Animal Experimentation Ethic Committee of Khon Kaen University.

In vivo experimental protocol: All rats were randomly assigned to 5 groups of 8 animals each.

Group I Vehicle+AF64A: Rats had been treated with vehicle for 1 weeks before and 1 week after the administration of AF64A, a cholinotoxin, in order to induce a cholinergic deficit as found in Alzheimer's disease.

Group II Donepezil+AF64A: Animals were treated with Donepezil, a cholinesterase inhibitor which used as standard drug for dementia treatment that served as positive control in this study, as same as that mentioned in group II.

GroupIII-V Mulberry fruits+AF64A: Rats had been treated with the plant extract at various doses ranging from 2, 10 and $50 \mathrm{mg} \mathrm{kg}^{-1}$ BW for 1 week before and 1 week after the administration of AF64A respectively (The doses used in this study were selected based on our preliminary data on the cognitive enhancing effect. In addition, all rats were received mulberry powder treatment at 1 month after the mulberry powder preparation).

The animals were determined the spatial memory 1 week after AF64A administration.

Then, they were sacrificed and determined the density of survival neurons and in various subregions of hippocampus.

AF64A administration: AF64A was prepared as an aqueous solution of acetylethylcholine mustard $\mathrm{HCl}$ (Sigma, St. Louis, MO) was adjusted to $\mathrm{pH} 11.3$ with $\mathrm{NaOH}$. After stirring for $30 \mathrm{~min}$ at room temperature, the $\mathrm{pH}$ was lowered to 7.4 with the gradual addition of $\mathrm{HCl}$ and stirred for $60 \mathrm{~min}$. The amount of AF64A was then adjusted either to $2 \mathrm{nmol} 2 \mu \mathrm{L}^{-1}$. The vehicle of AF64A was distilled water prepared in the same manner as the AF64A and recognized as ACSF. In order to administer AF64A bilaterally via intracerebroventricular (i.c.v.) route, the animals were anesthetized with the intraperitoneal injection of sodium pentobarbital at dose of $60 \mathrm{mg} \mathrm{kg}^{-1} \mathrm{BW}$. Then, AF64A ( $\left.2 \mathrm{nmol} 2 \mu \mathrm{L}^{-1}\right)$ was infused bilaterally via intracerebroventricular (i.c.v.) route with a 30-gauge needle inserted through a burr hole drilled into the skull into both the right and left lateral ventricles. Stereotaxic coordinates were (from the bregma): posterior $0.8 \mathrm{~mm}$, lateral $\pm 1.5 \mathrm{~mm}$ and ventral (from dura) $3.6 \mathrm{~mm}$. The rate of infusion was $1.0 \mu \mathrm{L} \mathrm{min}{ }^{-1}$ and the needle was left in place for 5 min after infusion and then slowly withdrawn.

Morris water maze test: The Morris water maze test is one of the most important paradigms used for testing spatial navigation task, which is thought to be dependent on the proper functioning of the hippocampus. The testing apparatus for all task used in this study was a stainless steel circular pool that $147 \mathrm{~cm}$ in diameter and $47 \mathrm{~cm}$ in dept. The interior of the pool was flat and the pool was placed on the steady floor. The pool was filled with water to a depth of $12 \mathrm{~cm}$. The water were maintained at $23 \pm 1^{\circ} \mathrm{C}$ and darkened by nontoxic powder.

The pool was divided into four quadrants (NE, NW, SE and SW) by two imaginary lines crossing the center of the pool. For each animal, the invisible platform was placed in the center of one of the quadrants and was remained there for a training period of 4 days. Each rat was gently placed in the water facing the wall of the pool from one of the four starting points (N, E, S or W) along the perimeter of the pool and the animal was allowed to swim until it climbed onto the platform. When an animal could not reach the platform in $60 \mathrm{~s}$, it was gently placed on the platform by the experimenter. In either case, the animal was left on the platform for $10 \mathrm{~s}$ and removed from the pool. Then, it was quickly dried with a towel before being returned to the home cage. The behavior of the experimental animal such as latency to finding the platform, total distance traveled, time spent in the target quadrant of the pool were recorded.

Histological procedure: Following anesthesia with sodium pentobarbital ( $\left.60 \mathrm{mg} \mathrm{kg}^{-1} \mathrm{BW}\right)$, fixation of the brain was carried out by transcardial perfusion with fixative solution containing $4 \%$ paraformaldehyde in $0.1 \mathrm{M}$ phosphate buffer $\mathrm{pH}$ 7.3. The brains were removed after perfusion and stored over a night in a fixative solution that used for perfusion. Then, they were infiltrated with $30 \%$ sucrose solution for approximately $4^{\circ} \mathrm{C}$. The specimens were frozen rapidly and $30 \mu \mathrm{M}$ thick sections were cut on cryostat. They were rinsed in the phosphate buffer and picked up on slides coated with $0.01 \%$ of aqueous solution of a high molecular weight poly L-lysine.

Morphological analysis: Five coronal sections of each rat in each group were studied quantitatively. Neuronal 
Am. J. Applied Sci., 9 (9): 1503-1511, 2012

counts in hippocampus were performed by eye using a 40x magnification with final field $255 \mu \mathrm{m}^{2}$ according to the following stereotaxic coordinates: AP $-4.8 \mathrm{~mm}$, lateral $\pm 2.4-6 \mathrm{~mm}$, depth 3-8 $\mathrm{mm}$. The observer was blind to the treatment at the time of analysis. Viable stained neurons were identified on the basis of a stained soma with at least two visible processes. Counts were made in five adjacent fields and the mean number extrapolated to give total number of neurons per $255 \mu^{2}$. All data are represented as number of neurons per $255 \mu \mathrm{m}^{2}$.

Determination of malondialdehyde level and acetylcholinesterase activity: Hippocampus was isolated and prepared as hippocampal homogenate and the Determination of the Malondialdehyde (MDA) level and acetylcholinesterase activity in hippocampus were performed. Malondialdehyde was indirectly estimated by determining the accumulation of Thiobarbituric Acid Reactive Substances (TBARS) while the activity of AChE was determined using.

Statistical analysis: Data are presented as mean \pm Standard Error of Mean (S.E.M). One-way Analysis of Variance (ANOVA), followed by Tukey post hoc test. A probability level less than 0.05 was accepted as significance.

\section{RESULTS}

The current results demonstrated that mulberry fruit (Morus alba var Chiangmai) contained high amount of anthocyanin, a subclass of flavonoid and phenolic compounds. Within 1 year at $-20^{\circ} \mathrm{C}$, the freeze dried mulberry powder exhibited the reduction of anthocyanin and phenolic compounds approximate 26.73 and $21 \%$ respectively. Within 1 month after the mulberry fruit powder preparation, the powder still showed strong antioxidant activity and the ability to suppress AChE activity. Mulberry fruit treatment could suppress AChE activity resulting in the enhanced spatial memory at the same magnitude as standard drug or Acetylcholinesterase Inhibitor (AChI) used nowadays. Moreover, mulberry fruit also decreased oxidative stress damage in hippocampus and gave rise to the increased neuron density in the mentioned area.

Quantification of phenolic compounds and anthocyanin: The results were shown in Table 1. Our results showed that the total phenolic compounds of mulberry fruit powder used in this study was $519 \pm 0.004$ $\mathrm{mg} \mathrm{GAE} / \mathrm{g}$ fresh matter of fruit. However, the content of phenolic compounds decreased to $410.28 \pm .001 \mathrm{mg}$ $\mathrm{GAE} / \mathrm{g}$ fresh matter of fruit or approximate $21 \%$ when kept in the dark air tight bottle at $-20^{\circ} \mathrm{C}$.
Table 1: Total phenolic compounds and anthocyanin content of mulberry fruit powder

\begin{tabular}{lll}
\hline $\begin{array}{l}\text { Materials/ duration } \\
\text { time }\end{array}$ & $\begin{array}{l}\text { Total phenolic compound mg } \\
\text { GAE /g fresh matter of fruit }\end{array}$ & $\begin{array}{l}\text { Anthocyanin mg CGE } \\
/ 100 \text { of fresh weight }\end{array}$ \\
\hline 1month & $519.33 \pm 0.004$ & $634.56 \pm 23.62$ \\
1 year & $410.28 \pm 0.001$ & $551.06 \pm 5.90$ \\
Percentage change & $21 \%$ & $13.17 \%$ \\
\hline
\end{tabular}

Table 2: The scavenging ability of mulberry powder and Vitamin C. $* \mathrm{p}<0.05$ as compared with vitamin C. ** $\mathrm{p}<0.01$ as compared with vitamin C. $* * * \mathrm{p}<0.001$ as compared with vitamin C

\begin{tabular}{lllll}
\hline & DPPH $(\mathrm{mmol}$ TEAC/100g FW) & FRAP activity $\left(\mathrm{mmol} \mathrm{Fe}^{2}+/ 100 \mathrm{~g} \mathrm{FW}\right)$ \\
$\begin{array}{l}\text { Concentration } \\
\text { (ug/mg) }\end{array}$ & vitamin & $\begin{array}{l}\text { Mulberry } \\
\text { fruity powder }\end{array}$ & $\begin{array}{l}\text { vitamin } \\
\text { C }\end{array}$ & $\begin{array}{l}\text { Mulberry } \\
\text { fruity powder }\end{array}$ \\
\hline 25 & $36.13+0.61$ & $17.63 \_0.2$ & $2.30 \pm 0.01$ & $5.86+0.03$ \\
50 & $40.75+0.2$ & $22.83+0.61$ & $10.90 \pm 0.1$ & $9.7+0.130$ \\
100 & $44.80+0.2$ & $32.95+2.04$ & $31.52 \pm 0.27$ & $18.86+0.74$ \\
250 & $52.60+1.63$ & $52.95+2.25$ & $53.24 \pm 0.20$ & $28.76+0.24$ \\
500 & $63.87+0.20$ & $64.74+1.63$ & $77.71 \pm 1.62$ & $59.29+0.24$ \\
1000 & $86.71+0.82$ & $82.66+1.63$ & $98.29 \pm 0.13$ & $95.24+0.67$ \\
\hline
\end{tabular}

Since anthocyanin pigment is recognized as a subclass of flavonoid, one types of phenolic compounds commonly found in mulberry fruits and playing the crucial role on learning and memory, we also determined the total anthocyanin content of mulberry fruit powder used in this study. Our results showed that the anthocyanin content assessed at 1 month after the mulberry powder preparation was $634.56 \pm 23.62 \mathrm{mg}$ of Cyanidin-3-Glucoside Equivalents (CGE) per $100 \mathrm{~g}$ of fresh weight. However, the content of anthocyanin decreased to $551.06 \pm 5.90 \mathrm{mg}$ of Cyanidin-3-Glucoside Equivalents (CGE) per $100 \mathrm{~g}$ of fresh weight $(13.17 \%$ reduction) within 1 year at $-20^{\circ} \mathrm{C}$ as shown in Table 1 .

Determination of antioxidant activity of mulberry fruits powder: It has been well established that the principle of antioxidant activity is the availability of electrons to neutralize free radicals. Since, antioxidant mechanisms are diverse, a variety of in vitro technique has been developed. It is suggested that the evaluation of antioxidant activity should be performed via different assays based on different mechanisms. Therefore, in this study we determined the antioxidant activity of mulberry fruit powder using DPPH and FRAP. Table 2 showed the scavenging ability of mulberry powder and Vitamin $\mathrm{C}$. The results showed that at concentration of 25,50 and $100 \mu \mathrm{g} \mathrm{mL}^{-1}$, vitamin $\mathrm{C}$ significantly exhibited DPPH scavenger efficacy ( $<<0.03$; compared to vitamin C). However, when the concentrations of both substances were increased further to 250,500 and $1000 \mu \mathrm{g} \mathrm{mL}^{-1}$, no significant changes in DPPH scavenger efficacy between vitamin $\mathrm{C}$ and mulberry fruit powder were observed. Our results showed that at concentration of $25 \mu \mathrm{g} \mathrm{mL} \mathrm{m}^{-1}$, mulberry powder exhibited higher ferric reducing antioxidant power compared to vitamin $\mathrm{C}$ ( $\mathrm{p}<0.0002$; compared to vitamin $\mathrm{C}$. Therefore both DPPH and FRAP assays also 
confirmed the antioxidant activity of mulberry fruit powder. Previous study reported that there was a strong relationship between polyphenol and reducing power (Sofidiya et al., 2006). Therefore the antioxidant activity of mulberry fruit observed in this study might be associated with its polyphenolic compound content.

In vitro assessment of $\mathrm{AChE}$ activity: The hypofunction of cholinergic system has been reported to play a crucial role on the cognitive impairment in both aged human and rodents (Papandreou et al., 2006), therefore, we also investigated the effect of mulberry fruit powder on the activity of AChE. The effect of mulberry fruit powder was shown in Fig. 1. It was found that mulberry fruit powder also demonstrated the suppression of AChE activity in a dose-dependent manner. Previous study had demonstrated that the principal role of $\mathrm{AChE}$ is the termination of acetylcholine (ACh), an important neurotransmitter at the cholinergic synapse (Mukherjee et al., 2007) resulting in the increase concentration of $\mathrm{ACh}$ at the synapse leading to the increased nerve impulse transmission (Rollinger et al., 2004). Our data showed that mulberry fruit powder exhibited the suppression of $\mathrm{AChE}$ at low concentration. Therefore, it should have the potential to enhance learning and memory.

The neuroprotective and cognitive enhancing effects of mulberry fruit: Based on the antioxidant activity of mulberry fruit and the role of phenolic compound including anthocyanin on memory, we have also evaluated the neuroprotective effect of mulberry fruit on the hippocampal neuron density, the area contributing the important role on learning and memory. Figure 2 demonstrated the effect of various doses of mulberry fruit powder on the neuron density in various subregions of hippocampus, the area playing the crucial role on learning and memory. Donepezil treated group significantly enhanced neuron density in
CA1 ( $<<0.01$ and .001 respectively; compared to vehicle+AF64A), CA2 ( $<<0.001$ all; compared to vehicle+AF64A), CA3 CA2 ( $<<0.001$ all; compared to vehicle+AF64A) and dentate gyrus $(\mathrm{p}<.001$ and .05 respectively; compared to vehicle+AF64A). Rats subjected to mulberry fruit powder treatment at dose of $2 \mathrm{mg} \mathrm{kg}^{-1}$ BW significantly enhanced neuron density only in CA1 and CA3 ( $<<.05$ all; compared to vehicle+AF64A). The medium dose or mulberry fruit at dose of $10 \mathrm{mg} \mathrm{kg} \mathrm{BW}$ could produce the significant increase in neuron density in CA1 $(\mathrm{p}<0.01$; compared to vehicle+AF64A), CA3 $(\mathrm{p}<0.01 ;$ compared to vehicle+AF64A) and dentate gyrus $(\mathrm{p}<0.05$; compared to vehicle+AF64A). Unfortunately, no change was observed at the higher dose.

Based on the crucial role of oxidative stress mentioned earlier, the level of MDA which previously reported to be the marker of oxidative damage was also determined in hippocampus. Figure 3 clearly revealed that both Donepezil and mulberry fruits at all doses of mulberry fruits could decrease MDA level in hippocampus $\quad(p<0.01 \quad$ all; compared to vehicle+AF64A). Taken all data together, the neuroprotective effect of mulberry fruit powder observed in this study might occur partly via the decreased oxidative stress damage indicating by the decreased MDA level.

Since mulberry fruit powder exhibited the neuroprotective effect in hippocampus, the cognitive enhancing effect of this substance was also determined and results were demonstrated in Fig. 4 and 5. It was found that both Donepezil and mulberry fruit powder treated groups at all dosage range used in this study significantly decreased escape latency time as shown in Fig. 4 ( $p<0.001$ all; compared to vehicle+AF64A). In addition, Fig. 5 showed that Donepezil and mulberry fruit treated groups at doses of 2 and $50 \mathrm{mg} \mathrm{kg}^{-1} \mathrm{BW}$ also produced significant elevation in retention times $(\mathrm{p}<0.05$ all; compared to vehicle+AF64A).

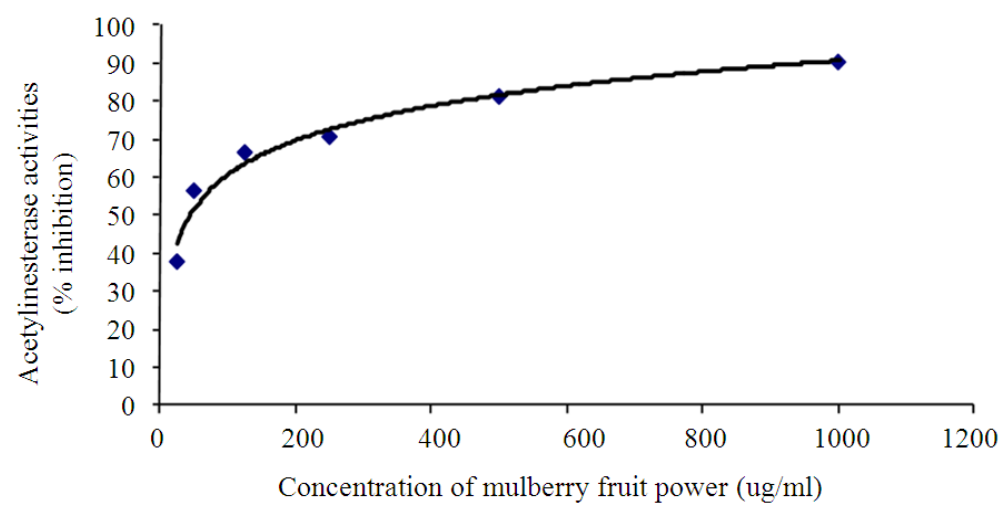

Fig. 1: The effect of mulberry fruit powder on the activity of AChE 


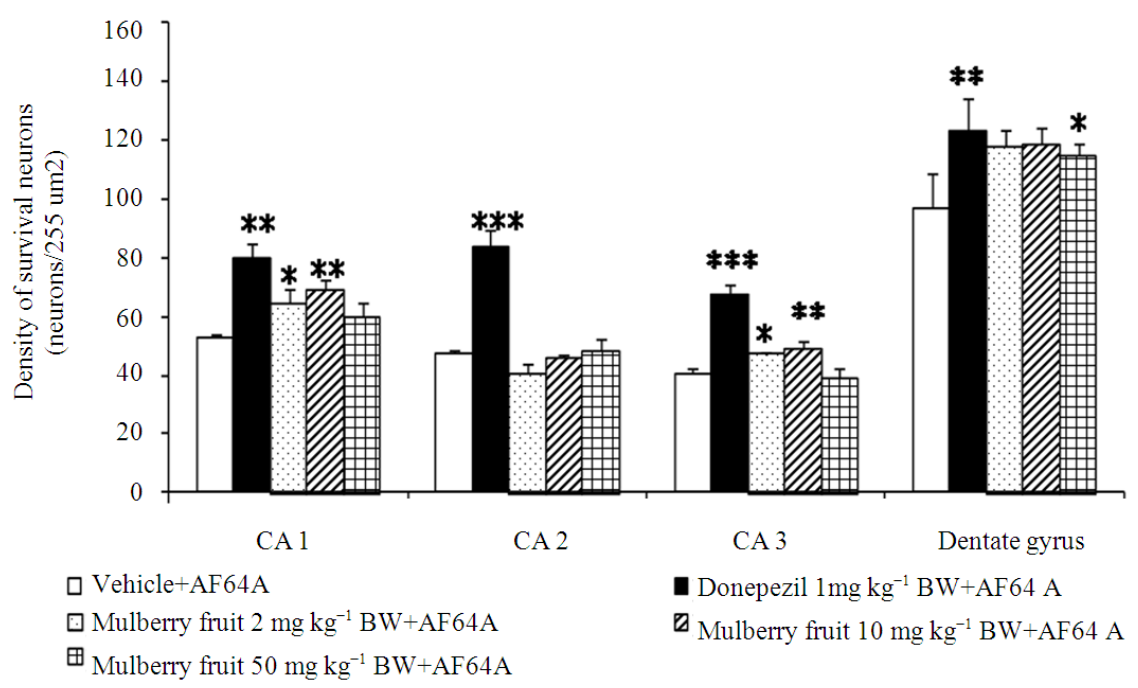

Fig. 2: The effect of mulberry fruit powder on the neuron density in various subregions of hippocampus. * p $<0.05$ as compared with vehicle plus AF64A. ** $\mathrm{p}<0.01$ as compared with vehicle plus AF64A. *** $\mathrm{p}<0.01$ as compared with vehicle plus AF64A

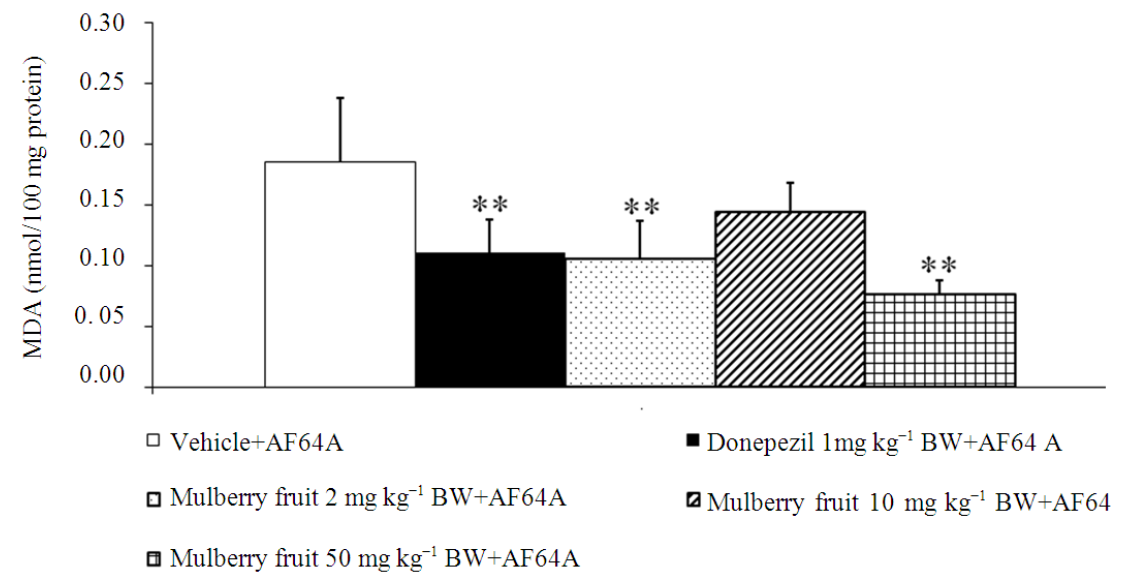

Fig. 3: The effect of mulberry fruit powder on the malondialdehyde (MDA) level in hippocampus.** $p<0.01$ as compared with vehicle plus AF64A

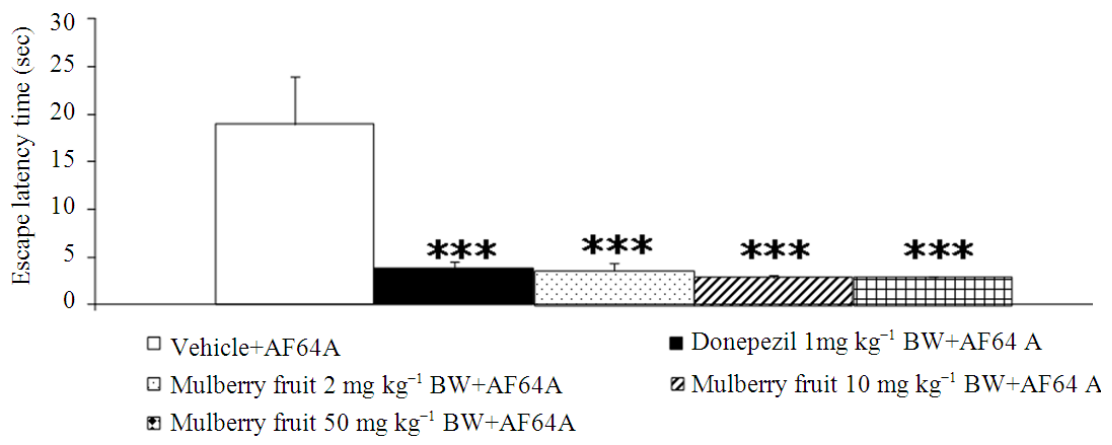

Fig. 4: The effect of mulberry fruit powder on the escape latency time in Morris water maze test. $* * * P$-value $<.01$ as compared with vehicle plus AF64A 


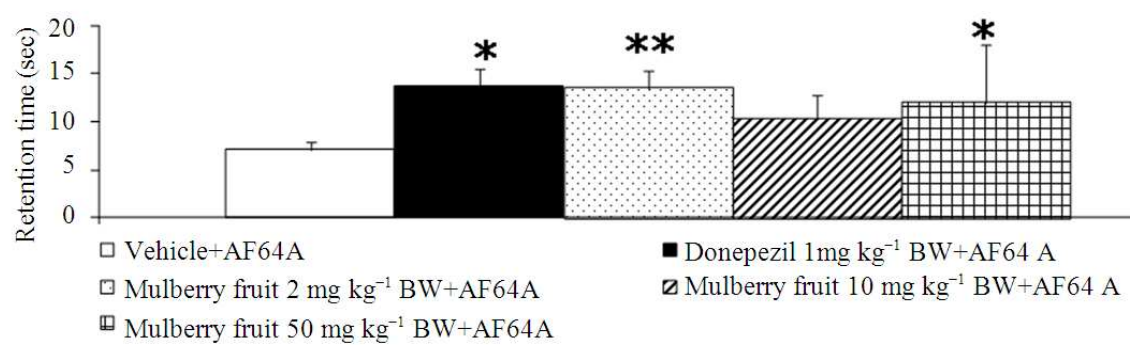

Fig. 5: The effect of mulberry fruit powder on the retention time in Morris water maze test. $* p<0.05$ as compared with vehicle plus AF64A. ** $\mathrm{p}<0.01$ as compared with vehicle plus AF64A

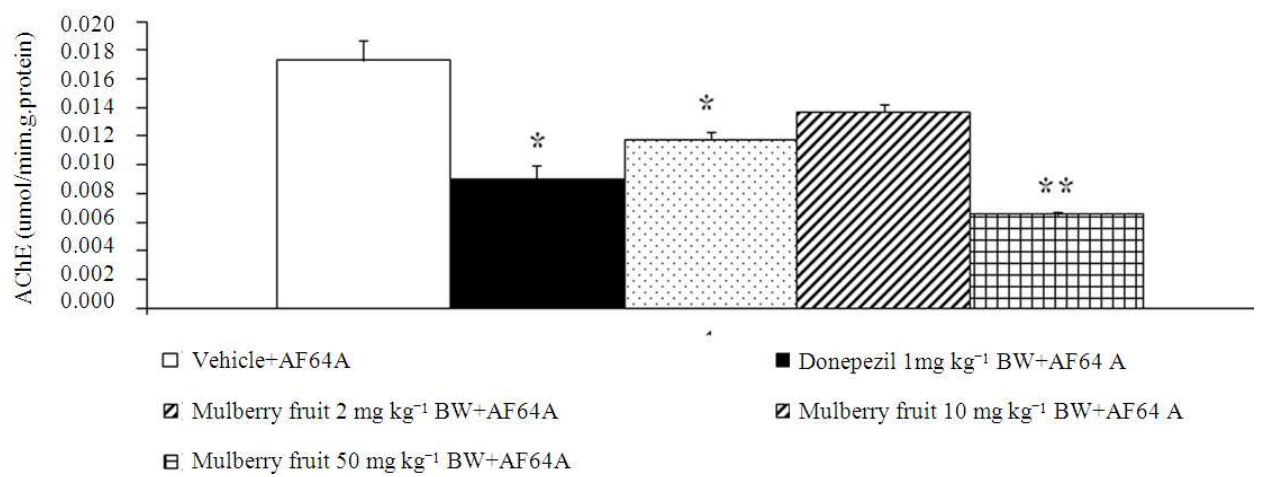

Fig. 6: The effect of mulberry fruit powder on the Acetylcholinesterase (AChE) activity in hippocampus. * p $<0.05$ as compared with vehicle plus AF64A. ** $\mathrm{p}<0.01$ as compared with vehicle plus AF64A

Although our in vitro study had demonstrated that mulberry fruit powder exhibited the AChE suppression effect, the difference environment between in vitro and in vivo might also influence on the response to mulberry fruit powder leading to the difference results. Therefore, in order to confirm that the suppression of AChE activity might play a crucial role on the cognitive enhancing effect of mulberry fruit, we also evaluated the activity of $\mathrm{AChE}$ in hippocampal homogenate at the end of the experimental period. The results were shown in Fig. 6. It was found that Donepezil produced a significant decrease in AChE activity in hippocampus. Rats subjected to mulberry fruit treatment at dose of 50 $\mathrm{mg} \mathrm{kg}^{-1}$ BW also significantly suppress AChE $(\mathrm{p}<0.01$; compared to vehicle+AF64A) while no significant changes were observed in other doses treatment. These results suggested that the cognitive enhancing effect might occur partly via the suppression of AChE. In addition, our data also suggested that besides the suppression of AChE activity, other factors such as the enhanced neuron density in hippocampus, the area contributing the important role on learning and memory, might also contribute their roles on the cognitive enhancing effect of mulberry fruit. However, the precise underlying mechanism is still required further investigation.

\section{DISCUSSION}

In this study, our results showed that AF64A treated rats induced the degeneration of neurons density in all subregions of hippocampus Previous work has demonstrated that hippocampus is regarded as a brain region essential for intact cognitive abilities and appears to be particularly vulnerable to the oxidative stress during aging (Fukui et al., 2002; Candelario-Jalil et al., 2001). The reduction of neuron number and the functional impairment in the mentioned area were reported to associate with oxidative damage. Increasing oxidative damage to proteins, lipids and nucleotides may contribute to neuron dysfunction in normal and pathological aging in humans (Ames et al., 1993; Harman, 1992). Recent study suggested that lipid peroxidation might be the potential therapeutic target in age-related cognitive decline (Montine et al., 2002).

Mulberry fruit powder exhibited the suppression of $\mathrm{AChE}$ activity in vitro and in hippocampus. In accompany with these changes, the memory impairment was also improved. In addition, Mulberry fruit powder treated group also showed the markedly reduction of MDA level in hippocampus. 
It is widely accepted that oxidative stress is implicated in the pathogenesis of neurodegeneration in numerous conditions including age-related cognitive decline (Stewart and Heales, 2003).

The reduction of MDA level observed in this study indicated the improvement of oxidative stress status which in turn resulted in the increased cholinergic neurons density leading to the attenuation of memory impairment. Previous study also clearly demonstrated that acetylcholinesterase inhibitor could also improve memory impairment in age-related cognitive decline. Therefore, the cognitive enhancing effect of Mulberry fruit powder in this study might occur partly via the inhibition of AChE.

\section{CONCLUSION}

Therefore, mulberry fruit is the potential cognitive enhancer and neuroprotectant against age-related cognitive decline. However, further investigation about the possible active ingredient is still essential.

\section{ACKNOWLEDGEMENT}

This study was supported by the Queen Sirikit Department of Sericulture, Ministry of Agriculture and Cooperatives, Thailand and The Integrative Complementary Alternative Medicine Research and Development Group, Khon Kaen University, Khon Kaen, Thailand.

\section{REFERENCES}

Ames, B.N., M.K. Shigenaga ans T.M. Hagen, 1993. Oxidants, antioxidants and the degenerative diseases of aging. Proce. Natl. Acad. Sci., 90: 7915-7922.

Benzie, I.F. and J.J. Strain, 1996. The Ferric Reducing Ability of Plasma (FRAP) as a measure of "antioxidant power": The FRAP assay. Anal. Biochem., 239: 70-76. PMID: 8660627

Butterfield, D.A., H.M. Abdul, S. Newman and T. Reed 2006. Redox proteomics in some age-related neurodegenerative disorders or models thereof. NeuroRx, 3: 344-357. DOI: 10.1016/j.nurx.2006.05.003

Candelario-Jalil, E., N.H. Mhadu, S.M. Al-Dalain, G. Martinez and O.S. Leo'n, 2001. Time course of oxidative damage in different brain regions following transient cerebral ischemia in gerbils. Neurosci. Res., 41: 233-241. PMID: 11672836
Ellman, G.L., K.D. Courtney, V Andres Jr and R.M. Feather-Stone, 1961. A new and rapid colorimetric determination of acetylcholinesterase activity. Biochem. Pharmacol., 7: 88-95. PMID: 137265187

Forster, M.J., A. Dubey, K.M. Dawson, W.A. Stutts and H. Lal et al., 1996. Age-related losses of cognitive function and motor skills in mice are associated with oxidative protein damage in the brain. Proc. Natl. Acad. Sci. USA, 93: 4765-4769. PMID: 8643477

Fukui, K., N.O. Omoi, T. Hayasaka, T. Shinnkai and S. Suzuki et al., 2002. Cognitive impairment of rats caused by oxidative stress and aging and its prevention by vitamin E. Ann. New York Acad. Sci., 959: 275-284. PMID: 11976202

Gong, S.X. and J.P. Zhu, 2008. Mulberry relieving nutritional anemi. J. Zhejiang Univ. Traditional Chinese Med., 32: 350-352.

Govoni, S., M. Amadio, F. Battaini and A. Pascale, 2010. Senescence of the brain: Focus on cognitive kinases. Curr. Pharm. Design, 16: 660-671. PMID: 20388076

Harman, D., 1992. Role of free radicals in aging and disease. Ann. New York Acad. Sci., 673: 126-141. DOI: 10.1111/j.1749-6632.1992.tb27444.X

Kang, T.H., J.Y. Hur, H.B. Kim, J.H. Ryu and S.Y. Kim, 2006. Neuroprotective effects of the cyanidin-3-O-beta-d-glucopyranoside isolated from mulberry fruit against cerebral ischemia. Neuroscie. Lett., 391: 122-126. PMID: 16181734

Kim, H.G., M.S. Ju, J.S. Shim, M.C. Kim and S.H. Lee et al., 2010. Mulberry fruit protects dopaminergic neurons in toxin-induced Parkinson's disease models. Br. J. Nutr., 104: 8-16. PMID: 20187987

Lutz, W., W. Sanderson and S. Scherbov, 2008. The coming acceleration of global population ageing. Nature, 451: 716-719. PMID: 18204438

Ma, Y.P., 2002. Clinical observation of mulberry as a medicine of pharyngitis treatment. Xinjiang $\mathrm{J}$. Traditional. Chinese Med., 20: 83-84.

Meda, A., C.E. Lamien, M. Romito, J. Millogo and O.G. Nacoulma, 2005. Determination of the total phenolic, flavonoid and proline contents in Burkina Fasan honey, as well as their radical scavenging activity. Food Chem., 91: 571-577. DOI: 10.1016/j.foodchem.2004.10.006

Mokrasch, L.C. and E.J. Teschke, 1984. Glutathione content of cultured cells and rodent brain regions: A specific fluorometric assay. Analytical Biochem., 140: 506-509. PMID: 6486436 
Montine, T.J., M.D. Neely, J.F. Quinn, M.F. Beal and W.R. Markesbery et al., 2002. Lipid peroxidation in aging brain and Alzheimer's disease. Free Radical Biol. Med., 33: 620-626. PMID: 12208348

Mukherjee, P.K., V. Kumar, M. Mal and P.J. Houghton, 2007. Acetylcholinesterase inhibitors from plants. Phytomedicine, 14: 289-300. PMID: 17346955

Murali, G. and C. Panneerselvam, 2007. Age-associated oxidative macromolecular damages in rat brain regions: Role of glutathione monoester. J. Gerontol. Series A: Biol. Sci. Med. Scie., 62: 824830. PMID: 17702872

Ning, D.W., B. Lu and Y.L. Zhang, 2005. The processing technology of mulberry series product. China Fruit Vegetables Process., 5: 38-40.

Papandreou, M.A., C.D. Kanakis, M.G. Polissiou, S. Efthimiopoulos and P. Cordopatis et al., 2006. Inhibitory activity on amyloid- $\beta$ aggregation and antioxidant properties of Crocus sativus stigmas extract and its crocin constituents. J. Agric. Food Chem., 54: 8762-8768. DOI: 10.1021/jf061932a

Pradham, S.N., 1980. Central neurotransmitters and aging. Life Sci., 26: 1643-1656.

Rollinger, J.M., A. Hornick, H. Langer, H. Stuppner and H. Prast, 2004. Acetylcholinesterase inhibitory activity of scopolin and scopoletin discovered by virtual screening of natural product. J. Med. Chem., 47: 6247-6254. PMID: 15566295

Scapagnini, G., C. Caruso and V. Calabrese, 2010. Therapeutic potential of dietary polyphenols against brain ageing and neurodegenerative disorders. Adv. Experimental Med. Biol., 698: 2735. PMID: 21520701
Shih, P.H., Y.C. Chan, J.W. Liao, M.F. Wang and G.C. Yen, 2010. Antioxidant and cognitive promotion effects of anthocyanin-rich mulberry (Morus atropurpurea L.) on senescence-accelerated mice and prevention of Alzheimer's disease. J. Nutrt. Biochem., 21: 598-605. PMID: 19443193

Shizhen, L., 2008. Compendium of Materia Medica. 1st Edn., Foreign Language Press, ISBN-10: 7119032607, pp: 4400.

Shukitt-Hale, B., F.C. Lau and J.A. Joseph, 2008. Berry fruit supplementation and the aging brain. J. Agric. Food Chem., 56: 636-641. PMID: 18211020

Sofidiya, M.O., A.O. Odukoya, O.B. Familoni and S.I. INya-Agha, 2006. Free radicals scavenging activity of some Nigerian medicinal plant extract. Pak. J. Biol. Sci., 9: 1438-1441.

Stewart, V.C. and S.J. Heales, 2003. Nitric oxideinduced mitochondrial dysfunction: implications for neurodegeneration. Free Radic Biol Med 2003; 34: 287-303. PMID: 12543245

Wrolstad, R.E., T.E. Acree, H. An, E.A. Decker and M.H. Penner et al., 2001. Current Protocols o in Food Analytical Chemistry. 1st Edn., John Wiley and Sons, New York, ISBN-10: 0471325651, pp: 1000 . 